\title{
¿ESTÁ PREPARADA NUESTRA ORGANIZACIÓN PARA HACER FRENTE ALOS RETOS DE LA INNOVACIÓN?
}

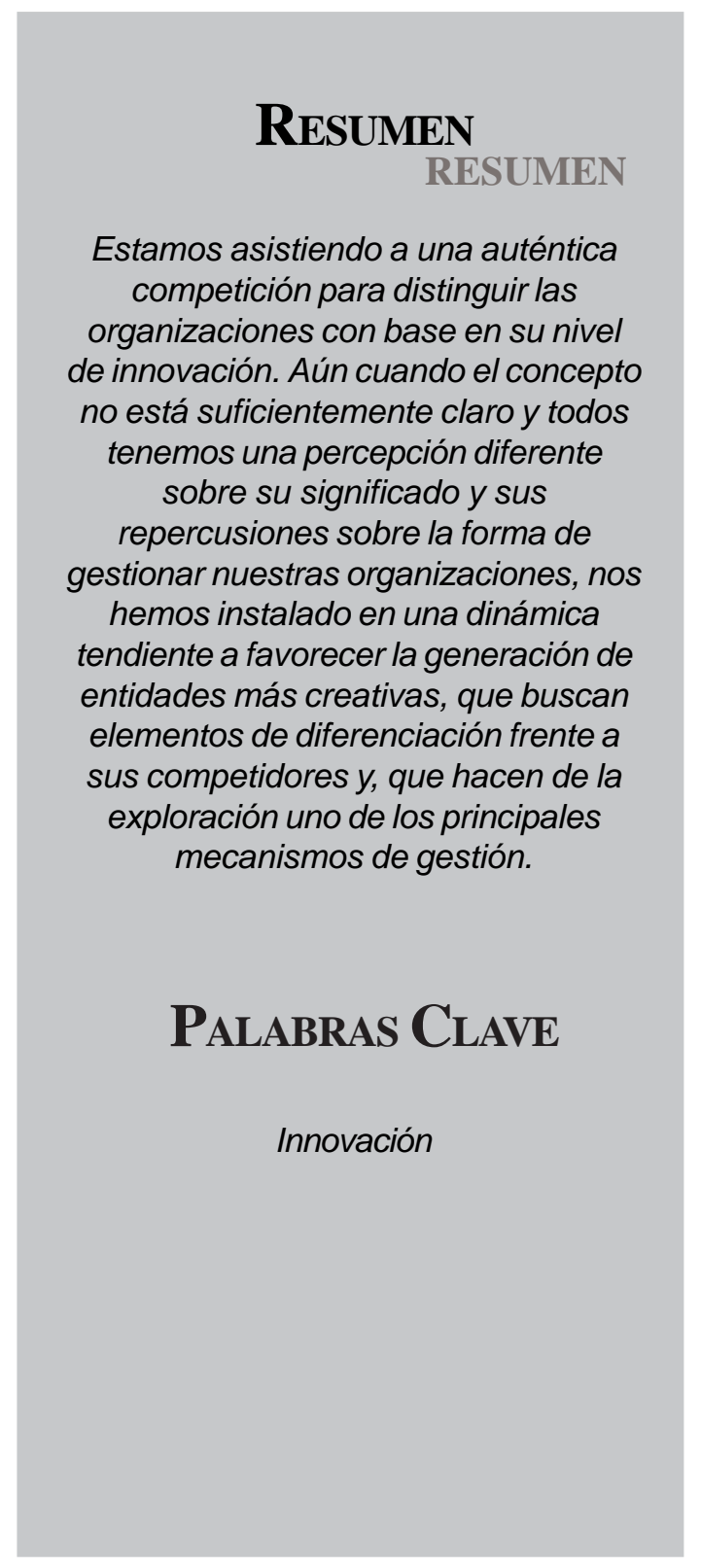

Sabin Azua*

$\mathbf{E}$ el Profesor Göran Roos (Socio de nuestra consultora $\mathrm{B}+\mathrm{I}$ Strategy), es la traslación del conocimiento en valor añadido. Esto lo sitúa en un plano diferente al de la Investigación y Desarrollo, que es la generación de conocimiento en base a los recursos aportados. Si esto es así, el ámbito de actuación de la Innovación se sitúa en todos los campos de vida de la organización, especialmente en el área de mercado.

Las organizaciones actuales se enfrentan a una serie de dinámicas competitivas que les fuerzan a desarrollar nuevos mecanismos de diferenciación frente a los competidores como único elemento favorecedor de la competitividad en el largo plazo: los mercados se han vuelto cada vez más globales, se han incrementado y diversificado los competidores (sobre todo los

\footnotetext{
* Socio Director de B+I Strategy.
} 
provenientes de nuevos entornos geográficos tradicionalmente «subdesarrolla-dos»), los clientes cada vez son más exigentes y dinámicos, la tecnología avanza a un ritmo acelerado, existe una fuerte competencia por la eficiencia operativa (los nuevos actores del mercado exigen una apuesta decidida por estos temas), las empresas cada vez más actúan en nodos de colaboraciones, etc. En síntesis, el talento y el conocimiento se convierten en activos cada vez más valiosos que el capital físico y financiero.

La búsqueda de empresas con capacidad de diferenciación frente a los competidores se da en todos los ámbitos de actuación de una empresa. Hay organizaciones que son excelentes en la diferenciación en el Qué (Productos, Servicios/Soluciones, Canales, Marcas, Experiencias de los Consumidores), otras lo son en el A Quién (Qué Clientes, En que Mercados, Qué Necesidades) y otros los son en el Cómo (Procesos, Alguna de las actividades de la Cadena de Valor, Redes y Alianzas, Modelo de Negocio). No hay reglas fijas, ni modelos, ni guías de actuación. Es lo verdaderamente apasionante del juego de gestión que nos ha tocado jugar.

Desde mi punto de vista, la innovación radical que requiere un número significativo de organizaciones occidentales pasa por la búsqueda de nuevos modelos de negocio orientados a la generación de un espacio de interacción con el mercado diferenciado del resto de los competidores. Las empresas deben saber combinar la gestión de la innovación incremental sobre los ámbitos de actuación tradicionales y, la preparación del futuro en base a una adecuada mezcla del qué, el cómo y el a quién para generar un modelo de negocio que ocupe el espacio del océano azul (Kim \& Mauborgne) que la empresa puede ocupar en el futuro.

Este hecho genera retos diferentes a la hora de gestionar nuestras organizaciones. Como

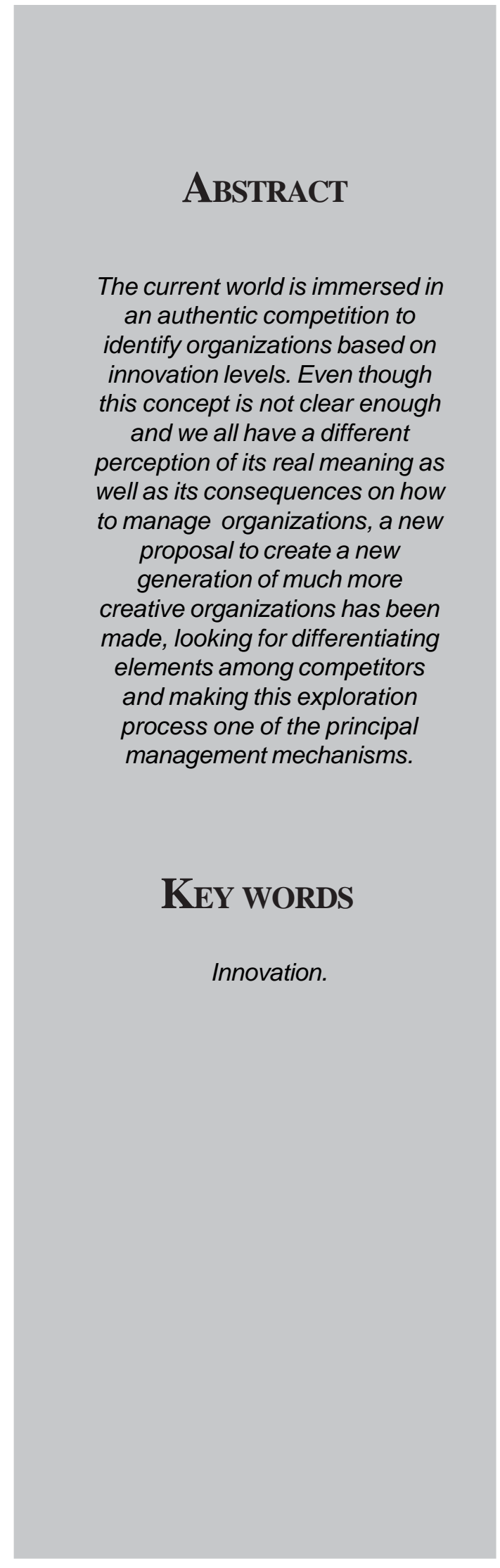

The current world is immersed in an authentic competition to identify organizations based on innovation levels. Even though is concept is not clear enough and we all have a different perception of its real meaning as well as its consequences on how manage organizations, a new proposal to create a new generation of much more creative organizations has been made, looking for differentiating elements among competitors and making this exploration process one of the principal management mechanisms.

\section{KEY WORDS} Innovation. 
establece Gary Hamel en su libro Liderando la Revolución:»hoy tenemos una nueva agenda de la innovación: deberá ser menos lineal en contraposición a la mejora continua, se centrará en conceptos empresariales y menos en productos y procesos, estará fuertemente orientada a la creación de riqueza, se generará en torno a las capacidades de la organización, requerirá activistas en la empresa y se hará en red con otras organizaciones». No cabe duda de que esto implica un nuevo reto para la gestión de nuestras organizaciones que hace necesario revisar la forma en que actualmente dirigimos.

La búsqueda de nuevos modelos organizacionales en los que las personas son realmente los principales actores de la competitividad de la empresa, donde el modelo empresarial y la forma de estructurar nuestras redes de colaboración e interacción con un número importante de agentes se convierte en determinante, donde la exploración de nuevos mercados no cubiertos por ningún actor del mercado es un motor de transformación de la organización, donde el papel educador y movilizador de los líderes es básico, etc., es el marco en el que debemos desarrollar nuestras capacidades para la innovación y la diferenciación frente a los competidores.

Ante este marco debemos reflexionar sobre el impacto del reto de la innovación sobre los diseños de las organizaciones. La arquitectura organizacional se convierte en un elemento relevante para el desarrollo de fuentes de ventaja competitiva y, al mismo tiempo, en una de las tareas fundamentales del equipo directivo. Hoy más que nunca los diseños organizativos son uno de los mecanismos de avance más poderosos que pueden desarrollar las organizaciones empresariales.

El problema es que muchas de las organizaciones actuales cuentan con un importante número de barreras para la innovación que deberán ser superadas en los diseños organizacionales y en la praxis diaria de las empresas, entre las que podríamos señalar: la excesiva presión del día a día y por los resultados a corto plazo, la excesiva focalización de las tareas de dirección en la reducción de costes y en la búsqueda a ultranza de la eficiencia, la poca implicación de la dirección en la innovación, la excesiva orientación a la «introspección» que desarrollan los equipos directivos, la obsesión por la competencia, la dificultad de «desaprender» de los esquemas de gestión del pasado, la existencia de culturas que no favorecen la creatividad ni tienen tolerancia con el error, etc.

En $\mathrm{B}+\mathrm{I}$ Strategy realizamos un proyecto de investigación sobre innovación estratégica «La Cometa de la Innovación» para identificar mecanismos de gestión de la innovación. Fruto de dicho proceso, generamos un modelo de innovación que se centraba en la superación de dichas barreras, intentando favorecer en las organizaciones una estrategia de innovación clara, una dinámica estructurada de trabajo para la innovación y una cultura de innovación que permita una actitud permanente de crea-tividad, exploración y búsqueda de nuevos espacios de innovación. Debemos procurar establecer claramente el desarrollo de negocios innovadores (sentido de dirección, cartera e productos/servicios innovadores, selección de proyectos de innovación) y, la generación de capacidad de innovación en la organización (liderazgo y cultura favorecedora de la innovación, gestión de las personas y de los talentos de la organización, establecer una adecuada dinámica de relaciones externas, tener una estructura organizativa flexible y orientada al cambio, gestión de procesos con un enfoque creativo y no de excesiva orientación a la eficiencia, selección de nuevos indicadores de gestión). Las organizaciones deberán generar contextos organizativos orientados hacia la innovación para hacer frente a los retos estratégicos que se señalaban al principio de este artículo. 
Para poder desarrollar esa dinámica organizativa tendente a favorecer la innovación en la empresa me gustaría proponer una serie de recomendaciones para el diseño organizacional:

a. Crea en las personas. No sé puede generar una dinámica de innovación en las organizaciones que no se sustente sobre las personas de la misma: atraiga personas excelentes y desarrollen buenos equipos, motive a las personas con nuevos retos y un proyecto de futuro interesante, déles foco, tiempo y recursos para pensar en el futuro y llevar adelante los nuevos proyectos, reconozca su valía y aportaciones, involucre al mayor número de personas en la innovación y busque la diversidad.

b. Piense en el futuro. Dedique tiempo a pensar en el futuro, genere mecanismos para que el día a día no absorba gran parte de su tiempo, aprenda a ver lo no convencional y sea un adicto a la novedad, relaciónese para pensar en el futuro, deje espacio para una gestión oportunista.

c. Apunte alto. Es preciso que se plantee objetivos ambiciosos, ya que sus aspiraciones fijan el límite superior de lo que es posible, plantee objetivos motivadores de forma que cada trabajador sienta que está contribuyendo a algo que marcará una diferencia genuina y positiva en las vidas de los clientes y compañeros.

d. Sea ágil. Es fundamental hacer participara los clientes, sea rápido en llevar al mercado las nuevas ideas, sea rápido en eliminar aquellas ideas que no funcionan, la meta es maximizar el ratio de aprendizaje por inversión, trate de beneficiarse de los efectos de la red y de los efectos positivos del feedback de los usuarios.

e. Deséelo con fuerza y diviértase. Hay que estar preparado par sortear barreras y resistencia al cambio, sea el paladín de la defensa de la necesidad de innovar, celebre con alegría los éxitos y alabe las lecciones aprendidas de los fracasos, disfrute de la faceta creativa de la innovación y comparta el entusiasmo por crear cosas nuevas.

Me gustaría animar a los directivos a convivir diariamente con estas cinco reglas para conseguir diseños organizativos que generen un contexto favorable a la innovación que permita afrontar con éxito el difícil reto de alcanzar empresas con capacidad de diferenciación frente a los competidores. 